\title{
EXPERIMENTAL STUDY OF VIBRATION EFFECTS ON HEAT TRANSFER DURING SOLIDIFICATION OF PARAFFIN IN A SPHERICAL SHELL
}

\author{
Johnathan J. Vadasz ${ }^{(1)}$, Josua P. Meyer ${ }^{(1)}$, Saneshan Govender ${ }^{(1,2)}$, Gennady \\ Ziskind $^{(3)}$ \\ ${ }^{(1)}$ Department of Mechanical and Aeronautical Engineering, University of Pretoria \\ e-mail: Johnathan@DrVadasz.com \\ Corresponding Author \\ ${ }^{(1)}$ e-mail: Josua.Meyer@up.ac.za \\ ${ }^{(1,2)}$ Gas Division EED, ESKOM \\ e-mail: Saneshan.Govender@eskom.co.za \\ ${ }^{(3)}$ Department of Mechanical Engineering, Ben-Gurion University \\ e-mail: gziskind@,bgu.ac.il
}

\begin{abstract}
Two effects that have been observed when metals and metal alloys are vibrated during solidification are a decrease in dendritic spacing, which directly affects density, and faster cooling rates and associated solidification times. Because these two effects happen simultaneously during solidification it is challenging to determine the one effect independently from the other. Most previous studies were on metals and metal alloys. In these studies, the one effect, i.e. the decrease in dendritic spacing, might influence the other, i.e. the faster cooling rates, and vice versa. The direct link between vibration and heat transfer has not yet been studied independently. The purpose of this study was to experimentally investigate the effect of vibration only on heat transfer and thus solidification rate. Experiments were conducted on paraffin wax, because it had a clearly defined macroscopic crystal structure consisting of mostly large straight-chain hydrocarbons. The advantage of the large straight-chain hydrocarbons was that the dendritic spacing was not affected by the cooling rate. Experiments were done with paraffin wax inside hollow plastic spheres of $40 \mathrm{~mm}$
\end{abstract}


diameter with $1 \mathrm{~mm}$ wall thickness. The paraffin wax was initially in a liquid state at a uniform temperature of $60^{\circ} \mathrm{C}$ and then submerged into a thermal bath at a uniform constant temperature of $15^{\circ} \mathrm{C}$, which was approximately $20^{\circ} \mathrm{C}$ below the mean solidification temperature of the wax. Experiments were conducted in approximately 300 samples, with and without vibration at frequencies varying from $10-300 \mathrm{~Hz}$. The first set of experiments were conducted to determine the solidification times. In the second set of experiments, the mass of wax solidified was determined at discrete time steps, with and without vibration. The results showed that paraffin wax had vibration independent of solid density contrary to other materials, eg. metals and metal alloys. Enhancement of heat transfer resulted in quicker solidification times and possible control over the heat transfer rate. The increase in heat transfer leading to faster solidifcation times was observed to first occur, as frequency increased and then to decrease.

KEYWORDS: solidification, sphere, frequency, vibration. 


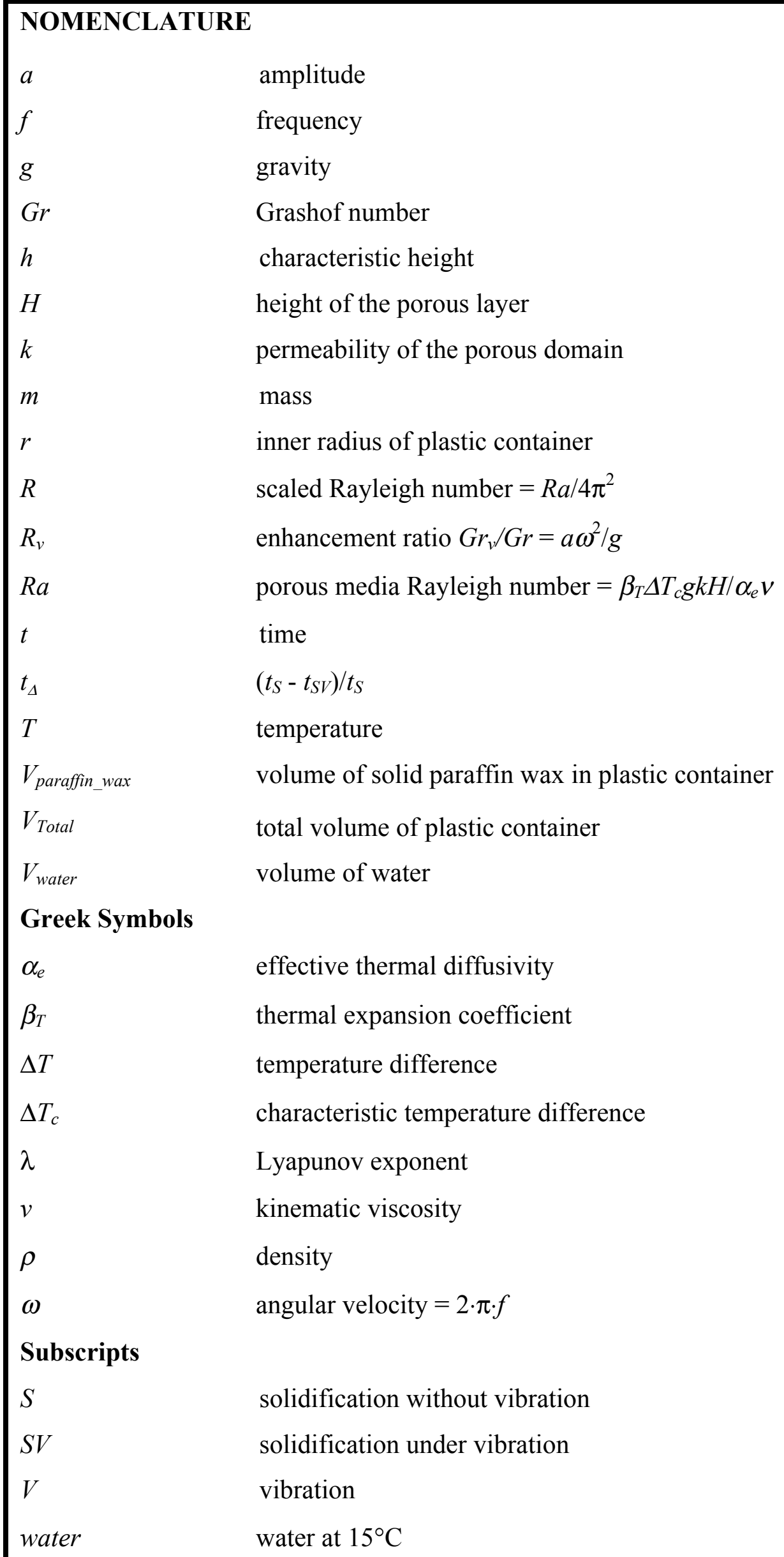

effective thermal diffusivity thermal expansion coefficient temperature difference characteristic temperature difference Lyapunov exponent kinematic viscosity density angular velocity $=2 \cdot \pi \cdot f$

solidification without vibration solidification under vibration vibration water at $15^{\circ} \mathrm{C}$ 


\section{INTRODUCTION}

The solidification of metals, such as binary alloys, has a wide range of applications because it constitutes the fundamental process in the production of such solid alloys. For a solidification process that occurs via planar solidification from a solid boundary, one may consider the presence of three distinct regions often identified as horizontal layers, i.e. a fluid binary mixture (the melt), the solid layer and a two-phase (fluidsolid) mushy layer, separating the other two.

The mushy layer is practically a porous medium consisting of an interconnected solid phase and having its voids filled with the melt binary fluid. Channelling in the mushy layer and the creating of freckles are considered the main reasons for nonhomogeneous solidification and the production of defects in the resulting solid product, as indicated by Peppin et al. [1]. The formation of defects adversely affects the mechanical properties of the solid product, leading to undesirable constraints on its industrial use.

It appears that ultrasonic vibration for metallurgical applications aiming at improving the cast metal quality dates back to 1878 , as indicated by Jian et al. [2]. This suggests that the "injection of ultrasonic energy into molten alloys brings about nonlinear effects such as cavitation, acoustic streaming, emulsification, and radiation pressure, which are used to refine microstructures, reduce segregation, and improve secondary phase formation and distribution".

In general, there is a large volume of both theoretical as well as experimental work attempting to determine the effect of vibrations on the solidification process. An attempt to link experimental results and theory was presented, for example, by Li et al. [3]. Mathematical modelling of convection-induced oscillatory structure formation in peritectic alloys was presented by Mazumder et al. [4]. This solved the convection equations numerically and predicted a band structure for the solid composition oscillating between two distinct values. Controlling the microstructure that results from the casting process is considered one of the main contemporary challenges of the foundry industry. Promoting fine microstructures in the cast is desirable because of its resulting favourable mechanical properties of strength, and ductility, as well as low susceptibility to microporosity and crack formation. 
Chirita et al. [5] indicate that mechanical, sonic and ultrasonic vibrations may promote "grain refinement, increased density, degassing, low shrinkage porosities, and changing the shape, size and distribution of the second phase". They investigated the influence of vibrations on aluminium-silicon alloys. Their experimental results show that mechanical properties were substantially affected by the level of applied frequency. Consequently, the tensile strength was improved for low vibration frequencies but decreased for high frequencies, as compared with gravity castings without vibration. Chirita et al. [5] mention that "a heat-transfer mechanism, that is acceleration dependent, seems to be responsible for the shift in mechanical properties' response to the vibration effect".

According to Jian et al. [2], "ultrasonic treatment of aluminium alloys, in general, has been studied extensively and it has been shown that the introduction of high intensity ultrasonic vibration into the melt can eliminate columnar dendritic structure, refine the equiaxed grains, and under some conditions, produce globular non-dendritic grains".

Mechanisms for grain refinement under ultrasonic vibrations have been proposed, for example, by Eskin [6] and Abramov et al. [7]. "They are related to ultrasonically induced cavitations, which produce large instantaneous pressure and temperature fluctuations in the melt. These pressure and temperature fluctuations are likely to induce heterogeneous nucleation in the melt. They are also likely to promote dendrite fragmentation by enhancing solute diffusion through acoustic streaming." Jian et al. [2], on the other hand, argue that there is no convincing evidence as to which mechanism, i.e. heterogeneous nucleation or dendrite fragmentation, is more important for grain refinement under ultrasonic vibrations.

Thus, previous work $[2,3,4,5,6,7]$ show two effects - a decrease in dendritic spacing and an increase in cooling rate with vibration during solidification. However, very limited experimental work if any was done on the effects of vibration during solidification on the cooling rate independent of any other effects. One such work (although numerical) was by Vadasz et al. [8] which studied the porous region under forced vibrations and the results showed that vibration influenced the heat transfer. 
Furthermore, because the numerical model was void of any metallurgical effects, such as dendritic spacing, it only considered the effects on heat transfer, or cooling rate, independently of any other effects. However, no experimental work has been done to verify this. Therefore, the purpose of this study is to experimentally study the vibration effects on heat transfer during solidification.

\section{EXPERIMENTAL METHODS AND PROCEDURE}

Paraffin wax, commercially referred to as RT35, was used as a medium on which to conduct experiments because it had a clearly defined macroscopic crystal structure consisting of mostly large straight-chain hydrocarbons. The advantage of the large straight-chain hydrocarbons was that the dendritic spacing was not affected by the cooling rate. The properties of the paraffin wax used were: a melting temperature of $29^{\circ} \mathrm{C}$ to $36^{\circ} \mathrm{C}$; a solidification temperature of $36^{\circ} \mathrm{C}$ to $31^{\circ} \mathrm{C}$ (typically $35^{\circ} \mathrm{C}$ ); with a density of $860 \mathrm{~kg} / \mathrm{m}^{3}$ at $15^{\circ} \mathrm{C}$; and with a flashpoint of $167^{\circ} \mathrm{C}$.

Experiments were conducted with spherical geometries because a comparison (although limited) could be made to work presented by Assis et al. [9], which studied the numerical and experimental solidification of paraffin wax, however, without vibration. One of the diameters studied was the same as in this study and therefore used for verification purposes. The spherical samples were generated from pouring liquid paraffin wax into a plastic container with an inner diameter of $40 \mathrm{~mm}$ and a wall thickness of $1 \mathrm{~mm}$. The geometries of the plastic container were measured with a vernier caliper.

Figure 1 shows the experimental set-up that was developed on which paraffin wax solidification experiments can be conducted, with and without vibration. The set-up consisted of a frame, a thermal bath with deionized water in the tank, a digital scale for weight measurements, a hot plate for melting of the paraffin wax, a data logger thermometer with K-type thermocouple for temperature measurements of the ambient air and molten paraffin wax, and an infrared thermometer to measure the temperature of the solidified paraffin wax.

Liquid paraffin wax, heated to $60^{\circ} \mathrm{C}$, was injected into the plastic spherical container. It was weighed to determine the mass and was suspended into the water in the thermal bath. The time from injection until it was placed into the water was less than 30 
seconds. As the ambient temperature was constant at $19^{\circ} \mathrm{C} \pm 2{ }^{\circ} \mathrm{C}$, the 30 seconds was negligible in comparison with the solidification time that was measured.

A thermal bath was used to cool the paraffin wax samples. The water temperature in the bath was maintained at $15^{\circ} \mathrm{C} \pm 0.1{ }^{\circ} \mathrm{C}$. The digital scale was accurate up to $1 / 100^{\text {th }}$ of a gram. The hot plate was a standard $1000 \mathrm{~W}$ kitchen appliance. A data logger thermometer was used with a thermocouple to measure the temperature of the paraffin wax with accuracy up to $0.1^{\circ} \mathrm{C}$. The infrared thermometer measured ambient temperature of $19^{\circ} \mathrm{C} \pm 2^{\circ} \mathrm{C}$.

The frame in Figure 1 was used to suspend the sample spheres inside the water tank of the thermal bath. The frame was also used to vibrate the samples by vibrating the cantilever beam, which suspended the samples. The cantilever beam was vibrated by a vibration exciter, which was placed underneath it and controlled by an amplifier and signal generator set at a frequency range between $10-300 \mathrm{~Hz}$. The vibration exciter had a pin, which vibrated by means of electromagnetic forces pulling it back and forth. To avoid any slippage of the pin at the contact point with the cantilever beam, a small recess was drilled into the beam where the pin rested. A seismometer computer program measured the frequency and amplitude of the sample sphere by means of an accelerometer sensor, where the amplitude was always $7.95 \mathrm{~mm} \pm 0.15 \mathrm{~mm}$.

Two different sets of experiments were conducted. In both sets, after the samples were cut into two halves they were photographed.

The first set of experiments had the paraffin wax completely solidify. The solidification time for samples, without vibration and with vibration at each frequency, was determined by iteration. The samples were cut into two pieces and the temperature of the paraffin wax was measured. When the temperature was measured at $15^{\circ} \mathrm{C} \pm 0.1^{\circ} \mathrm{C}$, the solidification time was recorded. The mass of the solidified paraffin wax was measured. The average density was also determined after the paraffin wax completely solidified.

The second set of experiments removed the samples at three time steps before the paraffin wax completely solidified. At each time step the sample was cut into two 
halves and the remaining liquid paraffin wax was poured out. The remaining paraffin wax was weighed and measurements of wall thickness and void size were recorded.

\section{DATA REDUCTION}

For the evaluation of the vibration effects on the solidification and thus heat transfer rate of the paraffin wax, three variables were needed. The first was the average density of the paraffin wax, the second was the Grashof number ratio $R_{v}$, and the third was the solidification time improvement $t_{\Delta}$.

After the liquid paraffin wax inside the plastic container was submerged in the thermal bath for a long enough period, the completely solidified paraffin wax samples were removed from the thermal bath. The average density of the paraffin wax completely solidified was then determined as:

$$
\rho_{\text {paraffin_wax }}=\frac{m_{\text {paraffin_wax }}}{V_{\text {paraffin_wax }}}=\frac{m_{\text {paraffin_wax }}}{V_{\text {Total }}-V_{\text {water }}}=\frac{m_{\text {paraffin_wax }}}{\frac{4}{3} \pi r^{3}-\frac{m_{\text {water }}}{\rho_{\text {water }}}}
$$

The mass of the completely solidified paraffin wax, $m_{\text {paraffin_wax, }}$ and water, $m_{\text {water, }}$, which was poured into sample spheres to fill the void, were determined using the digital scale. The density of water, $\rho_{\text {water, is }}$ known as $999.2 \mathrm{~kg} / \mathrm{m}^{3}$ [10] at a temperature of $15^{\circ} \mathrm{C}$.

The other variable that needs to be determined is the ratio between the vibration Grashof number $\left(G r_{V}=a \omega^{2} \beta_{T} \Delta T h^{3} / v^{2}\right)$, with vibration presented by Bardan and Mojtabi [11], and the Grashof number, $\left(G r=g \beta_{T} \Delta T h^{3} / v^{2}\right)$, without vibration at the paraffin wax.

$$
R_{v}=\frac{G r_{v}}{G r}=\frac{a \omega^{2}}{g}
$$

The enhancement ratio, $R_{v}$, of the Grashof numbers in Equation 2 takes into consideration the enhancement of vibration on the Grashof number, Gr. If this 
enhancement is more than one, then it shows that vibration affected the heat transfer during solidification. The rate of heat transfer, which is represented in a nondimensional form by the Nusselt number, is in general a function of the Reynolds number, Prandtl number and Grashof number. The Grashof number is in general the only non-dimensional group that is relevant in this study as it identifies a direct relation to the effects of vibration as presented in Equation 2 by the enhancement ratio. The enhancement ratio is directly proportional to the amplitude, $a$, and the square of the angular velocity, $\omega$, which is directly related to the square of the frequency of vibrations.

The solidification time improvement was determined as

$t_{\Delta}=\left(t_{S}-t_{S V}\right) / t_{S}$

where $t_{s}$ was the measured time to solidification without vibration, and $t_{s v}$ the time to solidifcation with vibration.

\section{RESULTS AND DISCUSSION}

The results from experiements on approximately 300 samples are presented in four parts. The first part is to determine the density of the solidified paraffin wax. The second part is to determine the solidification times. The third part is to determine the effects the vibrations had on the solidification time. The fourth part is to compare the results presented in the current study with the numerical results presented by Vadasz et al. [8]. 


\section{Densities}

The average densities of the samples versus frequency are shown in Table 1. It was found that for every sample of five experiments, the average densities of the experiments varied by less than $1 \%$. The first set of samples at a frequency of $0 \mathrm{~Hz}$ shows the results for no vibration. The other six sets of samples show the frequency ranges from $10 \mathrm{~Hz}$ to $300 \mathrm{~Hz}$. The results show that as the enhancement ratio increased from 0 to 461, the average densities remained constant. The average density of all samples in Table 1 was calculated to be $859.93 \mathrm{~kg} / \mathrm{m}^{3}$, and the highest deviation was $1.2 \%$ from this average. Therefore, for the purpose of this study it was deduced that the density of the solidified paraffin wax was a constant. This value compares well with the $860 \mathrm{~kg} / \mathrm{m}^{3}$ provided by the supplier of the paraffin wax.

\section{Solidification times}

The solidification time of samples without vibration is shown in Table 2 and with vibration in Table 3. From Table 2, it can be concluded that the paraffin wax solidified mass increased at each of the four time steps for all five samples without vibration, and Table 3, with vibration frequency of $100 \mathrm{~Hz}$.

Table 2 shows that all the samples of paraffin wax completely solidified at 30 minutes, and 25 minutes in Table 3. The experiments presented by Table 2 show the solidified paraffin wax was at $15^{\circ} \mathrm{C}$ at it's centre where the time of 30 minutes was recorded, and similarly, 25 minutes was recorded in Table 3. An iterative process of experiments led to these results. The $100 \mathrm{~Hz}$ frequency, shown in Table 3, was found to be the optimum frequency where the vibration had most affected the solidification time. This optimum frequency was used for all other experiments in the study. The solidification time result of 30 minutes without vibration shown in Table 2 compares well with the numerical and experimental results presented by Assis et al. [9], where the solidification time shown for $40 \mathrm{~mm}$ paraffin wax samples without vibration was approximately 36 minutes. The difference of 6 minutes may be due to the paraffin wax that was used by Assis et al. [9], having had a lower solidfication temperature than the one used in this study by a difference of about $7^{\circ} \mathrm{C}$. 
Effect of vibration on solidification times

Each one of the five sets of samples, from Table 2 and Table 3, is shown in Figure 2. The mass of the samples without vibration (Table 2) and the samples with vibration (Table 3) each varied by less than $1 \%$. Furthermore, the results show that the mass solidified with vibration was always higher then the mass solidified without vibration. The averaged results (in the last columns) from Table 2 and Table 3 are presented in Table 4.

It is shown that at 5 minutes, more solidified paraffin wax was formed under vibration comparing the $18.22 \mathrm{~g}$ with the $15.58 \mathrm{~g}$ under no vibration. This result shows a $2.64 \mathrm{~g}$ of extra paraffin wax that solidified with a vibration frequency of $100 \mathrm{~Hz}$ at 5 minutes, which is $17 \%$ higher than that without vibration. Table 4 is presented on a graph in Figure 3, which also shows how the line for solidification under vibration frequency of $100 \mathrm{~Hz}$ is always above that of the line representing no vibration for each time step above 0 minutes and below 25 minutes.

In Figure 3, the enhancement line shows the difference between the mass formed with and without vibration, presented in the last column of Table 4. The results from Table 4 show there was more heat transfer with vibration, and an overall 17\% improvement in solidification time calculated using Equation 3 and the final solidification times.

In Table 4 it is also shown how the enhancement decreased per time step, which is also clear from Figure 3 because as the two lines representing solidification under vibration and without vibration get closer. The final difference between the two solidified masses of $21.29 \mathrm{~g}$ and $21.00 \mathrm{~g}$, which is $0.29 \mathrm{~g}$, shown in Table 4 , is due to the initial amounts of liquid paraffin wax that were not exactly the same.

As the enhancement decays show in Table 4 and Figure 3, it never reaches zero. This means there is always more solidified paraffin wax formed under vibration than without. The significance of the decay may be attributed to the amount of liquid and solid in the sample. There are two suggested reasons for the decay. The first reason is because more solid was formed, the wall temperature had to be higher or equal than the thermal bath because it has only reached solid state, and also the liquid surface boundary was reduced, further decreasing heat transfer to the surroundings. The 
second considers the three layers during solidification, the solid, the liquid and the mushy layer. During solidification the solid layer increases, and thus the other two layers, the liquid and mushy layer, decrease. Since the effects of vibration happen in the liquid and mushy layer, it would make sense that as these layers get smaller the decay would also be affected.

Figure 4 shows the paraffin wax solidified after 5 minutes without vibration and with a vibration frequency of $100 \mathrm{~Hz}$. The outside blue line indicates the plastic sphere boundary, and the inside blue line indicates the void in the centre. The paraffin wax solidified between the two blue lines is shown, excluding the air gap at the top identified with a red line. Measurements of the average wall thickness of the solidified paraffin wax were taken and the results show approximately $2-3 \mathrm{~mm} \pm 0.1$ $\mathrm{mm}$ difference between the two samples, with the larger average wall thickness being that of the vibrated sample at $100 \mathrm{~Hz}$.

Figures 5 and 6 show the paraffin wax solidified after 10 minutes and 20 minutes without vibration and with a vibration frequency of $100 \mathrm{~Hz}$ respectivaly. Measurements of the average wall thickness of the solidified paraffin wax were taken and the results show a difference of approximately $2-2.5 \mathrm{~mm} \pm 0.1 \mathrm{~mm}$ after 10 minutes (Figure 5) and $1.5-2 \mathrm{~mm} \pm 0.1 \mathrm{~mm}$ after 20 minutes (Figure 6) between the two samples, with the larger average wall thickness being that of the vibrated sample at $100 \mathrm{~Hz}$.

The final solidification time shown in Figure 7 shows the samples after 30 minutes without vibration and 25 minutes with vibration frequency of $100 \mathrm{~Hz}$. It can be observed that although the void was still present in Figure 7 when there was vibration, it is very similar to the void formed without vibration. This is confirmed by the density calculations done in Table 1 . This means that the voids were geometrically of the same size.

The samples in Figures $4-7$ are one set of five done. The other four sets of experiments were done to check for repeatability. These results are shown in Table 2 and Table 3 for five samples each. 
Comparison with numerical results

The numerical results presented by Vadasz et al. [8] considered the vibration effects in a porous medium and were therefore used for comparison purposes. Figure 8 shows a graphical representation of the Lyapunov exponents as a function of the scaled Rayleigh number. A quantitative comparison of the experimental results of this study with the numerical work of Vadasz et al. [8] was not possible. The reason was that the numerical work solved the porous medium equations with vibration, whereas the current study considered the solidification process with vibration where the porous medium conditions could not be measured. However, qualitatively, a comparison could be made. The reason was that the effects of vibration were in the porous layer, as stated earlier. This meant that any effect in the porous layer would further affect the solidification process.

In Figure 8 (a), a chaotic orbit is observed at a scaled Rayleigh number when there is one or more positive Lyapunov exponents, a quasi-periodic orbit is observed when there is one negative Lyapunov exponent and two equal to zero, a periodic orbit is observed when there are two negative Lyapunov exponents and one equal to zero, and a fixed point is observed when all Lyapunov exponents are negative. Figure 8 (a) shows chaotic "bursts" at specific values of the scaled Raleigh number. This behaviour is quite distinct from the case without forced vibrations, when the chaotic solution occupies a wide range of scaled Rayleigh number values, interrupted only by periodic "bursts" in Vadasz et al. [8]. This result shows that the vibrations affect the heat transfer in a porous medium, yet what that effect is, is still to be determined. Figure 8 (b) shows a zoomed-in section of Figure 8 (a) for a smaller range of the scaled Rayleigh number. It can clearly be observed how many positive, negative and zero values are present in the Lyapunov exponents. Depending on the scaled Rayleigh number range of interest, a graph can be used to determine the exact values where a chaotic orbit changes to periodic, and back to chaotic.

In the current study, it was shown experimentally that the rate of solidification is increased when vibrations were introduced during the solidification process. Since the effects of vibration would only affect the porous section and/or liquid we can assume that the results presented by Vadasz et al. [8] in the porous medium might be linked to the results seen in the current study of solidification. It is also clear that vibrations do 
have an effect on the porous medium section as presented by Vadasz et al. [8] for Prandtl number in the range representing paraffin wax. Therefore, as a qualitative comparison, the results in the current study showing vibration having an effect on solidification may be due to the effects in the porous medium as seen in Vadasz et al. [8].

\section{CONCLUSIONS}

The direct link between vibration and heat transfer has not yet been studied independently. The purpose of this study was to experimentally investigate the effect of vibration only on heat transfer and thus solidification rate. Experiments were conducted on paraffin wax, because it had a clearly defined macroscopic crystal structure consisting of mostly large straight-chain hydrocarbons. The advantage of the large straight-chain hydrocarbons was that the dendritic spacing was not affected by the cooling rate.

It was found from experiments that the average densities of the paraffin wax samples were not affected by vibration during solidification. The average deviation between samples varied by less than $1.2 \%$, therefore, the density of the solidified paraffin wax was a considered constant. The experimental results indicated a decrease in solidification time of $17 \%$ (or increase in heat transfer) during solidification process with vibration when compared with to that without. This result was observed at an optimal vibration frequency of $100 \mathrm{~Hz}$. The decay in the increased rate of solidification under vibration could also be seen in this study. The decay shown by the decrease in mass enhancement from $17 \%$ down to $2 \%$ suggests a possible oscillatory decay. The enhancement ratio shown to increase from a value of $0-461$, with increase in frequency from $0-300 \mathrm{~Hz}$, presents no effects on the average solid density of the paraffin wax. Therefore, the current study has showed an independent relationship between the mechanical vibration properties and heat transfer (related to solidification time) during solidification, where the microstructure and material properties, such as dendritic spacing and denstiy, did not affect the cooling rate in the experiments. The numerical work referenced observed that vibration affected the porous media over a scaled Rayleigh number covering the range where paraffin wax 
would be. Qualititively, the comparsion with the numerical work obsereved that there was an effect on the solidification process due to vibration.

Further investigation will need to be conducted to understand better the effects seen in this paper and to look at the possible oscillatory decay or growth. More resolution in terms of the results, especially during the early parts of solidification, may give a better picture of how vibrations affect the cooling rate or solidification time.

\section{ACKNOWLEDGEMENTS}

The University of Pretoria and Prof JP Meyer gave much support for this project, both financial and intellectual. Also Ben-Gurion University, Prof G Ziskind and Prof A Levi provided much input into the project and supplied the equipment and facilities to run the project.

\section{REFERENCES}

1. S. S. L. Peppin, P. Aussillous, H. E. Huppert and M. Grae Worster, Steady-state mushy layers: experiments and theory, Journal of Fluid Mechanics, 570, 69-77, 2007.

2. X. Jian, H. Xu, T. T. Meek and Q. Han, Effect of power ultrasound on solidification of aluminum A356 alloy, Materials Letters, 59, 190-193, 2005.

3. M. Li, T. Tamura and K. Miwa, Controlling microstructures of AZ31 magnesium alloys by an electromagnetic vibration technique during solidification: from experimental observation to theoretical understanding, Acta Materialia, 55, 4635-4643, 2007.

4. P. Mazumder, R. Trivedi and A. Karma, A Model of Convection-Induced Oscillatory Structure Formation in Peritectic Alloys, Metallurgical and Materials Transactions A, 31A, 1233-1246, 2000.

5. G. Chirita, I. Stefanescu, D. Soares and F. S. Silva, Influence of vibration on the solidification behaviour and tensile properties of an $\mathrm{Al}-18 \mathrm{wt} \% \mathrm{Si}$ alloy, Materials and Design, 30, 1575-1580, 2009.

6. G. I. Eskin, Influence of cavitation treatment of melts on the processes of nucleation and growth of crystals during solidification of ingots and castings from light alloys, Ultrasonics Sonochemistry, 1 (1), S59-S63, 1994. 
7. V. Abramov, O. Abramov, V. Bulgakov and F. Sommer, Solidification of aluminium alloys under ultrasonic irradiation using water-cooled resonator, Materials Letters, 37 (1-2), 27-34, 1998.

8. J. Vadasz, J. P. Meyer and S. Govender, Chaotic and Periodic Natural Convection for Moderate and High Prandtl Numbers in a Porous Layer subject to Vibrations, Transport in Porous Media Journal, 103 (2), 279-294, 2014.

9. E. Assis, G. Ziskind and R. Letan, Numerical and Experimental Study of Solidification in a Spherical Shell, J. of Heat Transfer, 131 (2), 024502, 2008.

10. M. J. Moran, H. N. Shapiro, et al., Fundamentals of Engineering Thermodynamics $7^{\text {th }}$ edition, John Wiley\& Sons, Inc., 2011.

11. G. Bardan and A. Mojtabi, On the Horton - Rogers - Lapwood convective instability with vertical vibration: Onset of convection, Physics of Fluids, 12 (11), 2723-2731, 2000. 


\section{LIST OF TABLES:}

Table 1. Samples showing the relationship between the enhancement ratio $G r_{v} / G r$ and the average density of solidified paraffin wax. Every sample is the average of five samples.

Table 2. Measured mass of solidified paraffin wax at different times under no vibration for five samples.

Table 3. Measured mass of solidified paraffin wax at different times under a vibration frequency of $100 \mathrm{~Hz}$ for five samples.

Table 4. Average calculated mass of solidified paraffin wax at different times for no vibration and vibration at frequency of $100 \mathrm{~Hz}$. The results are the average results in the last columns of Tables 2 and 3. 


\section{LIST OF FIGURES:}

Figure 1: Picture and schematic of experimental set-up showing the thermal bath, vibration exciter, test sample location, and cantilever system attached to the rigid frame.

Figure 2: Mass of solid paraffin wax formed versus time for no vibration and vibration frequency of $100 \mathrm{~Hz}$ for five samples of each.

Figure 3: Average mass of solid paraffin wax formed versus time for no vibration and vibration frequency of $100 \mathrm{~Hz}$.

Figure 4: Solidified paraffin wax samples cut in half after 5 min for $0 \mathrm{~Hz}$ (no vibration) and $100 \mathrm{~Hz}$ (with vibration), with the blue outline indicating the average wall thickness of the solidified paraffin wax and the centre void, and the red outline indicating the air gap.

Figure 5: Solidified paraffin wax samples cut in half after $10 \mathrm{~min}$ for $0 \mathrm{~Hz}$ (no vibration) and $100 \mathrm{~Hz}$ (with vibration), with the blue outline indicating the average wall thickness of the solidified paraffin wax and the centre void, and the red outline indicating the air gap.

Figure 6: Solidified paraffin wax samples cut in half after $20 \mathrm{~min}$ for $0 \mathrm{~Hz}$ (no vibration) and $100 \mathrm{~Hz}$ (with vibration), with the blue outline indicating the average wall thickness of the solidified paraffin wax and the centre void, and the red outline indicating the air gap.

Figure 7: Solidified paraffin wax samples cut in half after $30 \mathrm{~min}$ for $0 \mathrm{~Hz}$ (no vibration) and 25 minutes for $100 \mathrm{~Hz}$ (with vibration), with the blue outline indicating the void geometry formed, and the red outline indicating the air gap.

Figure 8: (a) Lyapunov exponents $\left(\lambda_{1}, \lambda_{2}\right.$, and $\left.\lambda_{3}\right)$ plotted as a function of the scaled Raleigh number, $R$, for an angular velocity of $\omega=45 \mathrm{rad} / \mathrm{s}$, and (b) showing a zoomed-in section of the scaled Raleigh number, $R$, from 320 to 390 . 
Table 1.

\begin{tabular}{llll}
\hline Sample & Frequency $(\mathrm{Hz})$ & $R_{v}=\frac{G r_{V}}{G r}$ & $\begin{array}{l}\text { Average Density } \\
{\left[\mathrm{kg} / \mathrm{m}^{3}\right]}\end{array}$ \\
\hline 1 & 0 & 0 & 865.73 \\
2 & 10 & 0.51 & 862.45 \\
3 & 25 & 3.2 & 849.55 \\
4 & 100 & 51 & 858.19 \\
5 & 110 & 62 & 859.44 \\
6 & 130 & 86.6 & 857.64 \\
7 & 300 & 461 & 866.53 \\
\hline
\end{tabular}

Table 2.

\begin{tabular}{lllllll}
\hline $\begin{array}{l}\text { Sample } \\
\rightarrow\end{array}$ & 1 & 2 & 3 & 4 & 5 & Average \\
\hline $\begin{array}{l}\text { Time } \\
\text { (minutes) }\end{array}$ & Mass [g] & Mass [g] & Mass [g] & Mass [g] & Mass [g] & Mass [g] \\
\hline 5 & 15.45 & 15.65 & 15.61 & 15.52 & 15.67 & 15.58 \\
10 & 17.75 & 17.77 & 17.98 & 17.68 & 17.97 & 17.83 \\
20 & 19.88 & 19.84 & 19.90 & 19.68 & 19.70 & 19.80 \\
30 & 21.18 & 21.22 & 21.35 & 21.40 & 21.30 & 21.29 \\
\hline Initial & & & & & & \\
mass [g] & 21.18 & 21.22 & 21.35 & 21.40 & 21.30 & 21.29 \\
\hline
\end{tabular}

Table 3.

\begin{tabular}{lllllll}
\hline $\begin{array}{l}\text { Sample } \\
\rightarrow\end{array}$ & 1 & 2 & 3 & 4 & 5 & Average \\
\hline $\begin{array}{l}\text { Time } \\
\text { (minutes) }\end{array}$ & Mass [g] & Mass [g] & Mass [g] & Mass [g] & Mass [g] & Mass [g] \\
\hline 5 & 18.15 & 18.18 & 18.16 & 18.27 & 18.34 & 18.22 \\
10 & 19.10 & 19.01 & 19.05 & 18.95 & 18.99 & 19.02 \\
20 & 20.24 & 20.29 & 20.11 & 20.14 & 20.27 & 20.21 \\
25 & 21.06 & 21.09 & 20.86 & 21.05 & 20.94 & 21.00 \\
\hline Initial & & & & & & \\
mass [g] & 21.06 & 21.09 & 20.86 & 21.05 & 20.94 & 21.00 \\
\hline
\end{tabular}

Table 4.

\begin{tabular}{llll}
\hline Sample $\rightarrow$ & $\begin{array}{l}\text { No vibration } \\
0 \mathrm{~Hz}\end{array}$ & $\begin{array}{l}\text { Vibration at } \\
100 \mathrm{~Hz}\end{array}$ & Enhancement \\
\hline Time (minutes) & Mass [g] & Mass [g] & Mass [g] \\
\hline 5 & 15.58 & 18.22 & $2.64(17 \%)$ \\
10 & 17.83 & 19.02 & $1.19(7 \%)$ \\
20 & 19.80 & 20.21 & $0.41(2 \%)$ \\
25 & - & 21.00 (Solidified) & \\
30 & 21.29 (Solidified) & - & \\
\hline
\end{tabular}



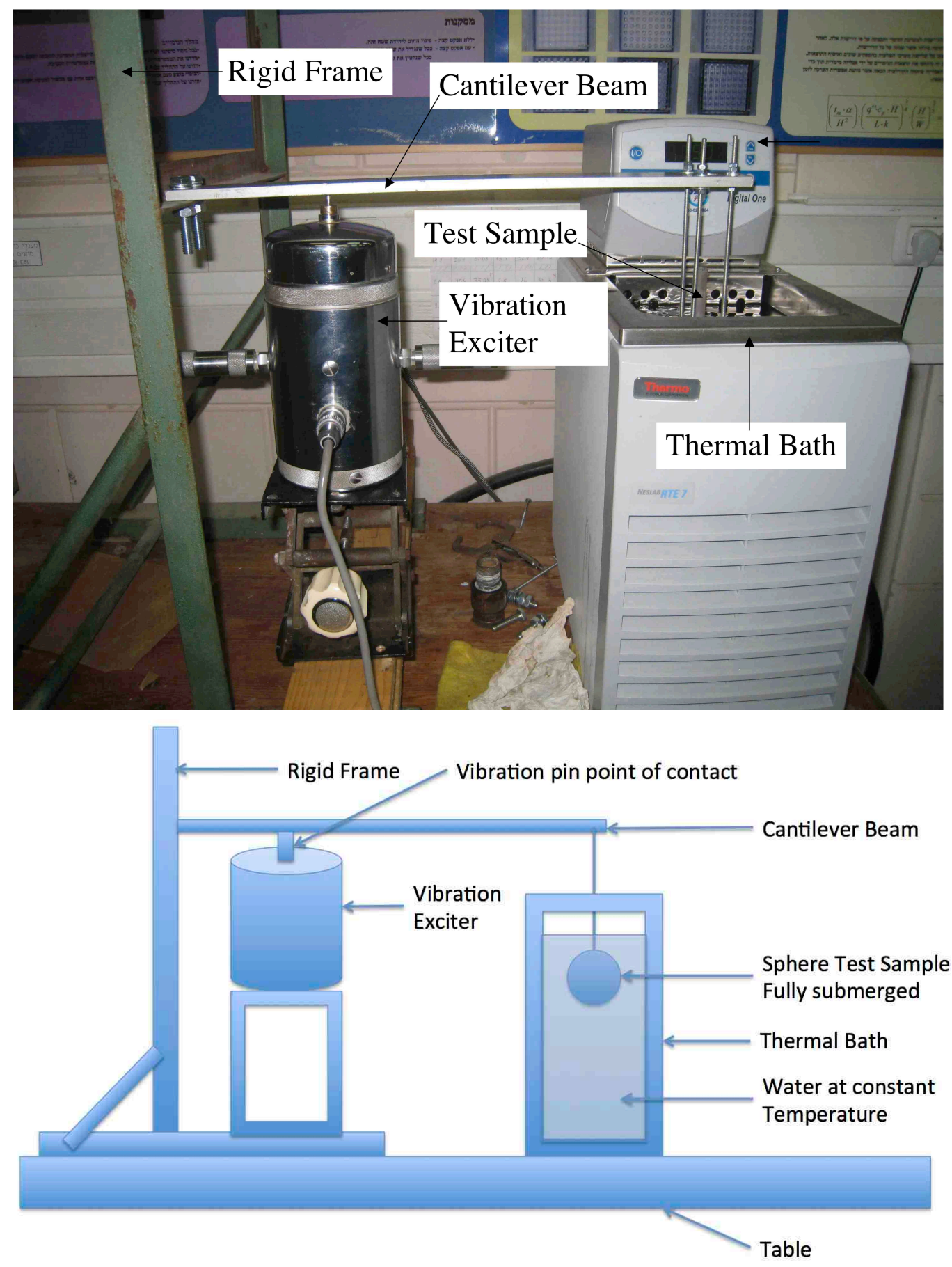

Figure 1 


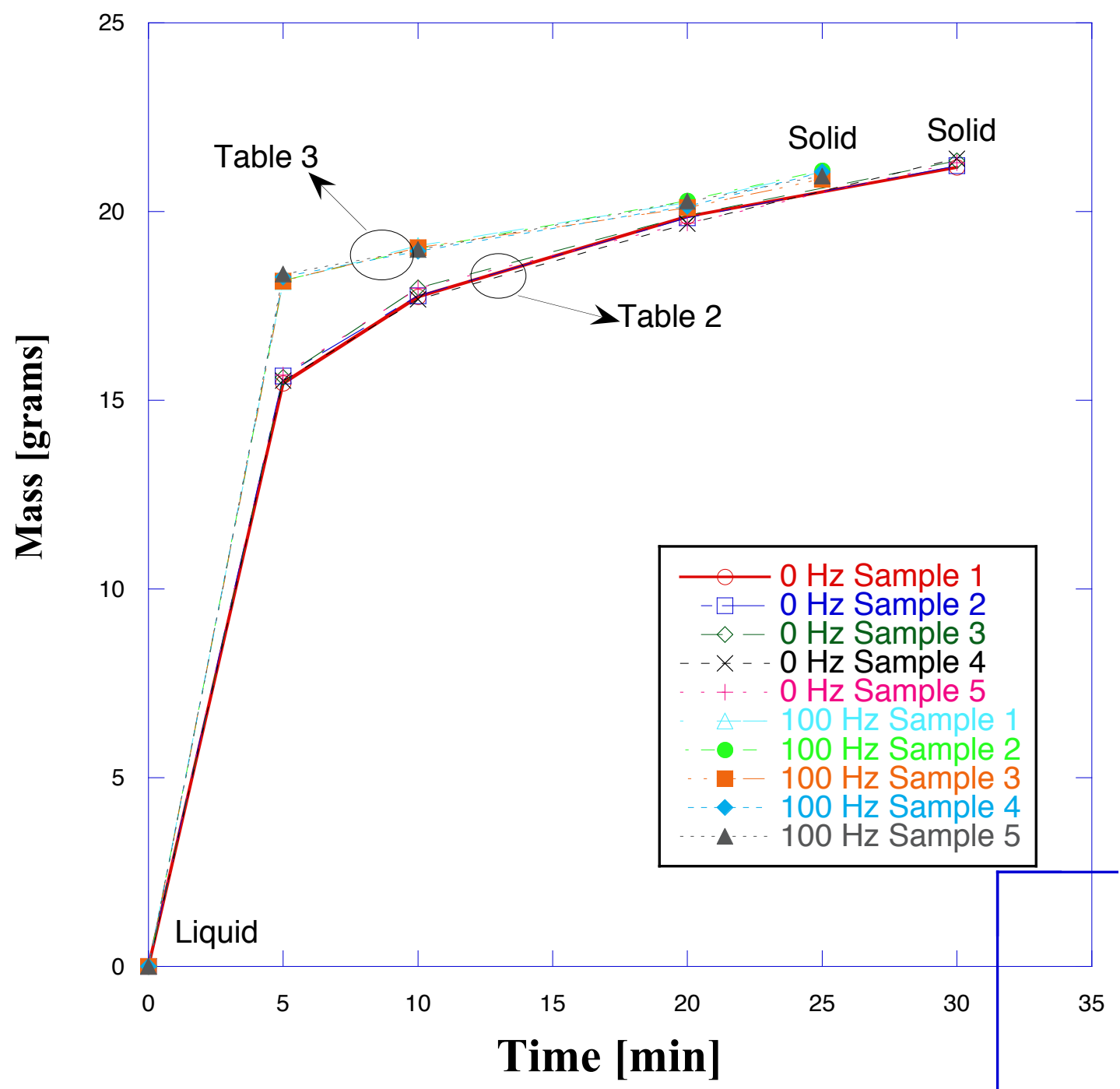

Figure 2 


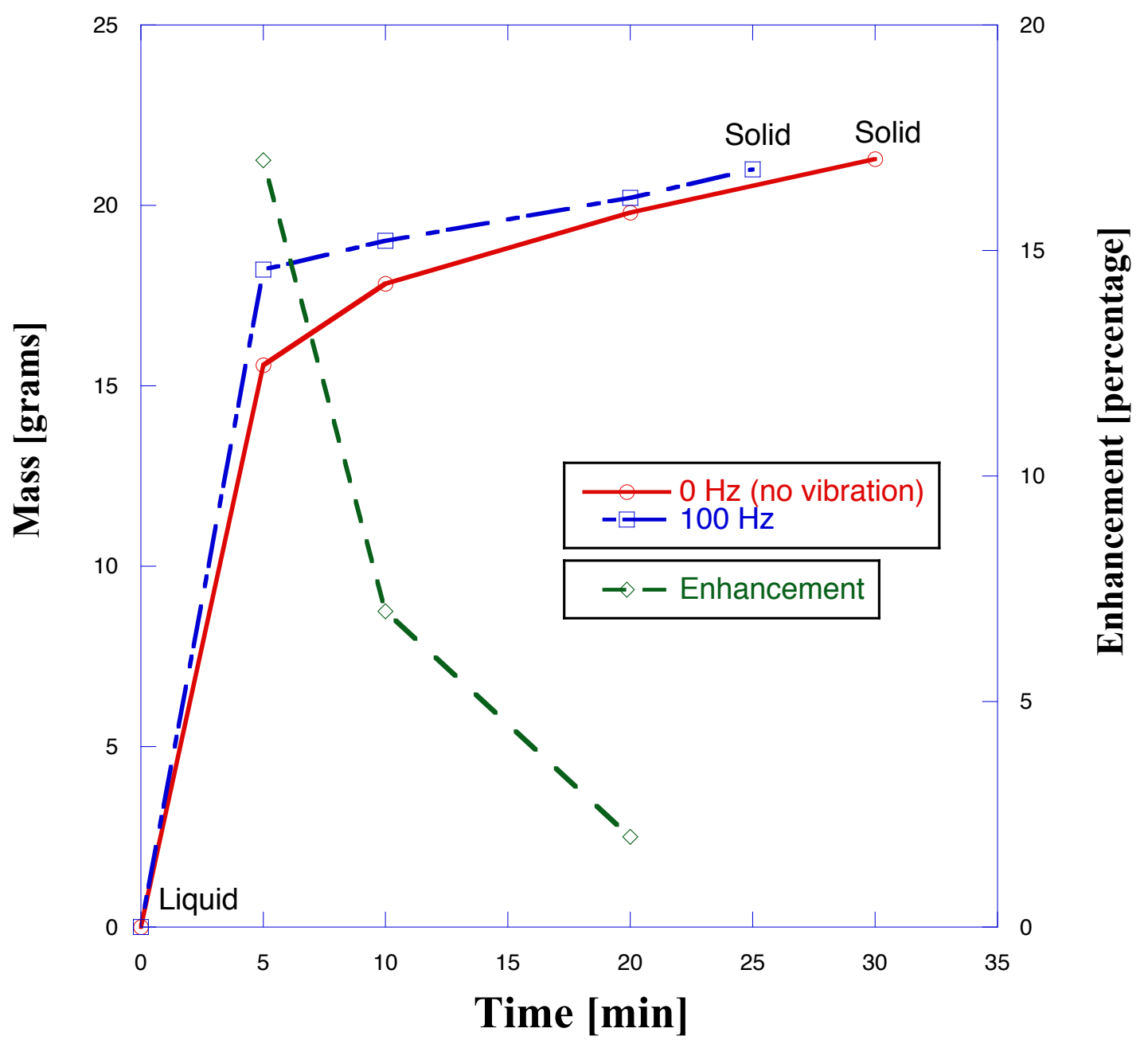

Figure 3 


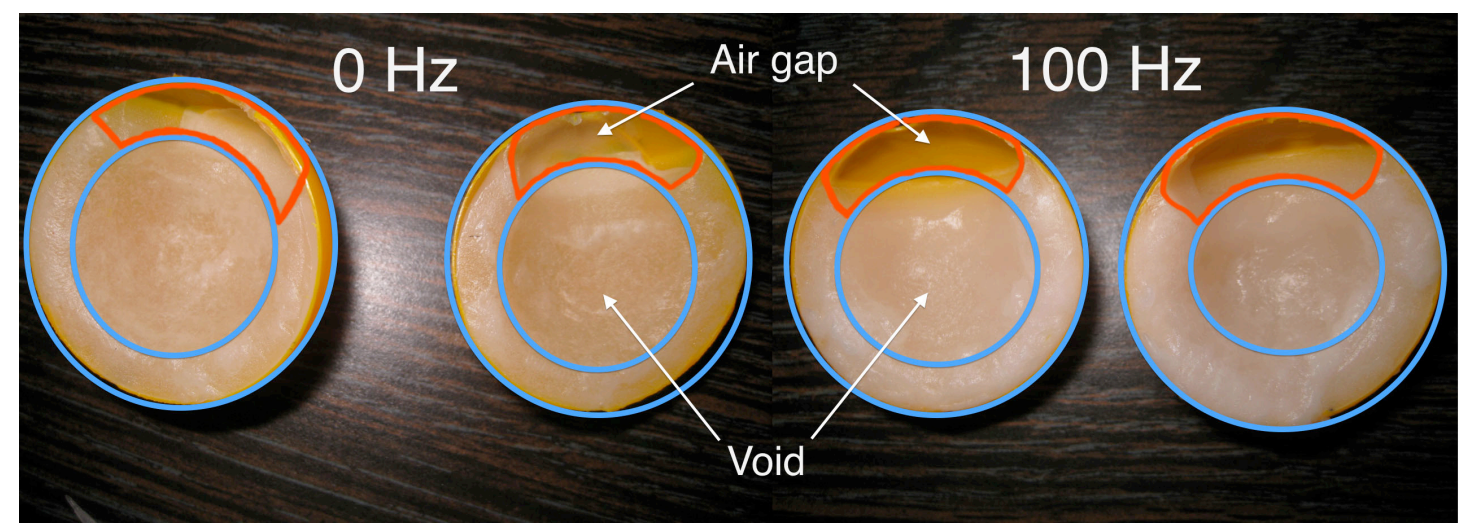

Figure 4

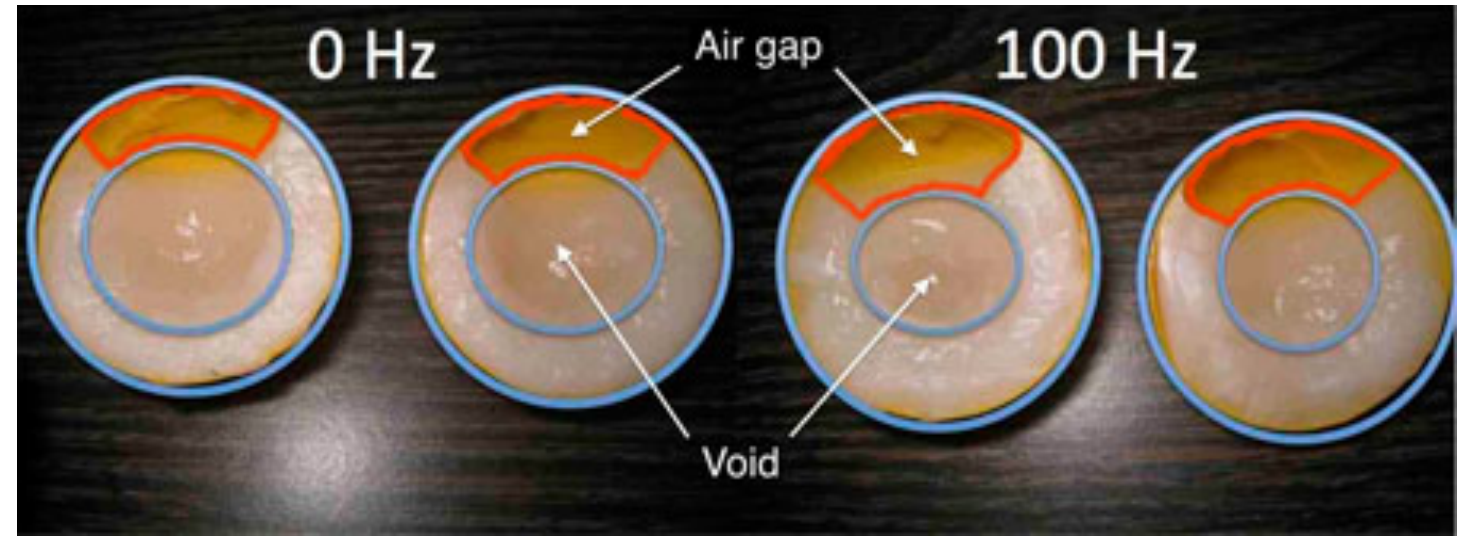

Figure 5

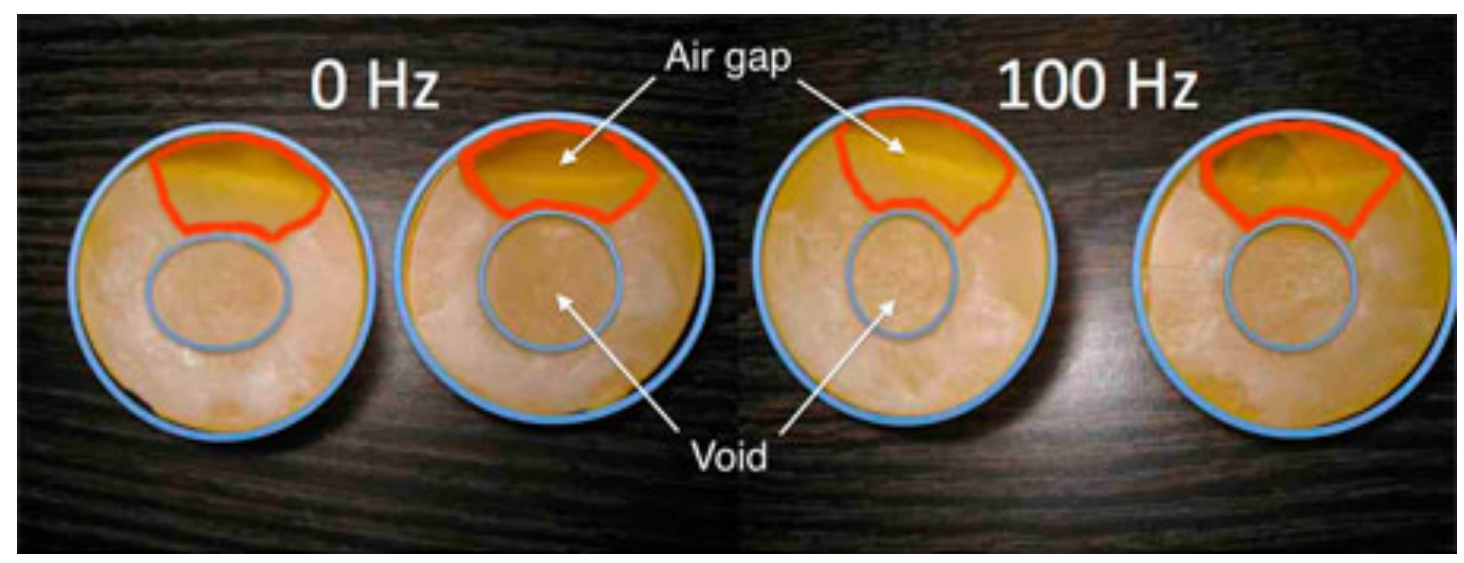

Figure 6

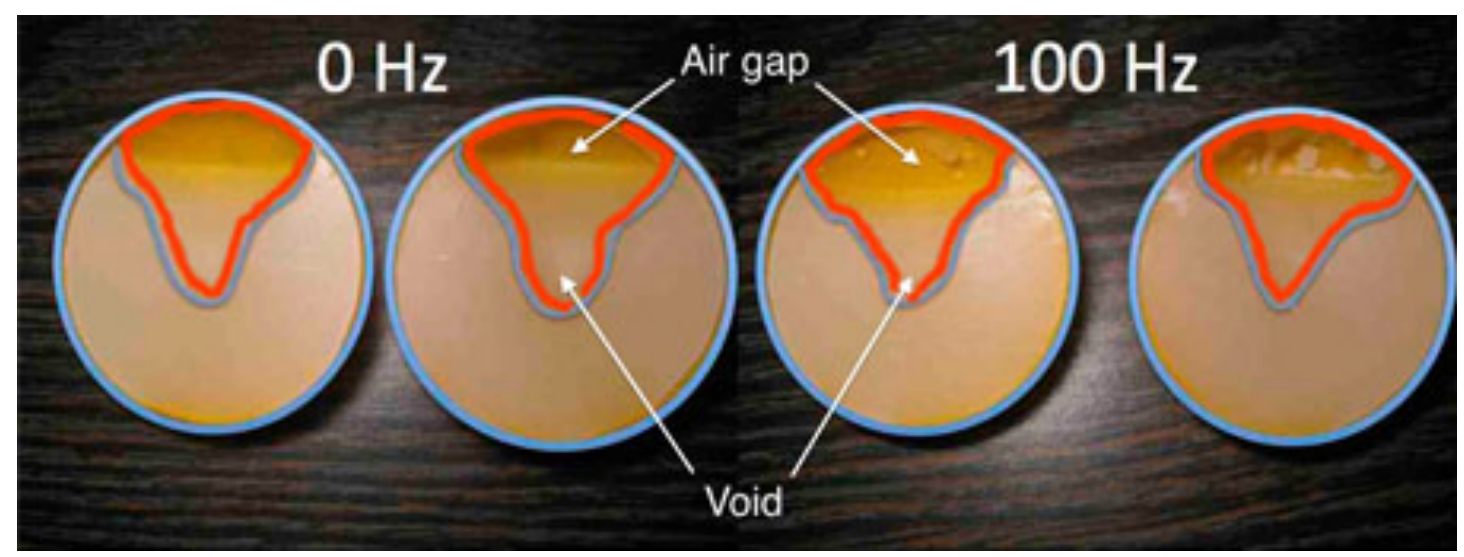

Figure 7 


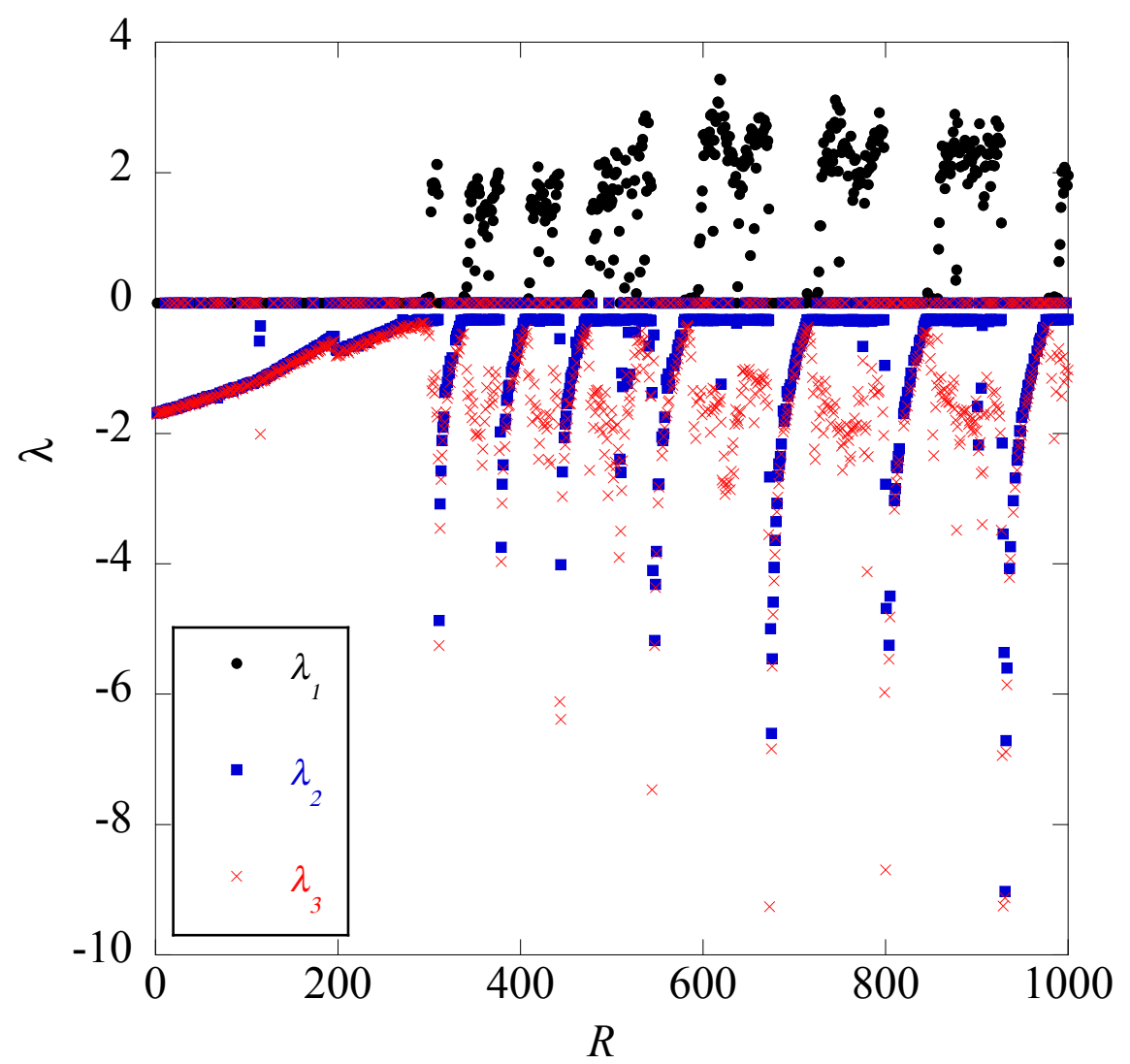

(a)

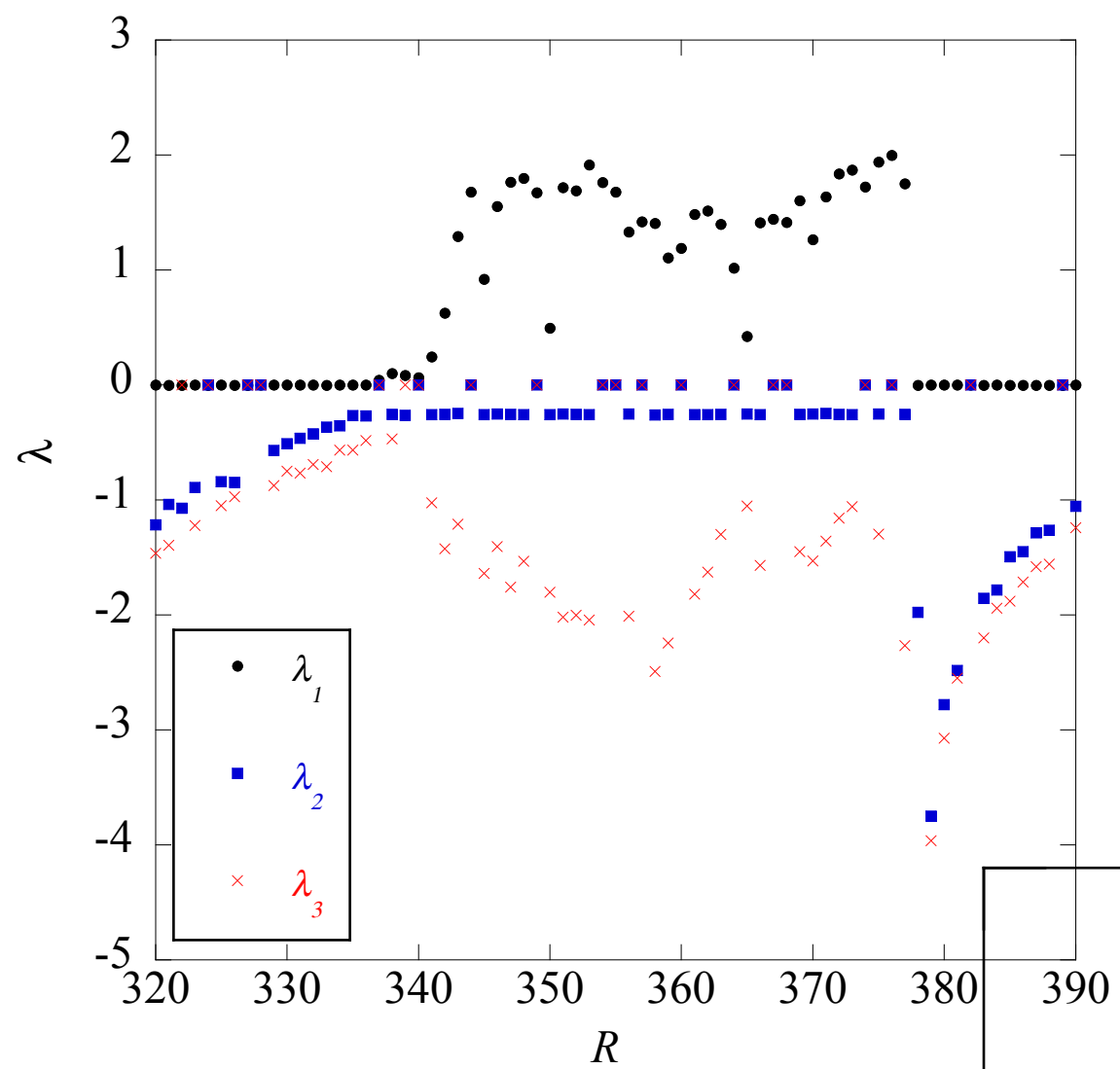

(b)

Figure 8 